\title{
El uso de fluoruros en niños menores de 5 años. Evidencia. Revisión bibliográfica.
}

\author{
María Gabriela Acosta de Camargo, ${ }^{1}$ \\ Lelimar Palencia, ${ }^{2}$ \\ Josnelly Santaella, ${ }^{2}$ \\ Liliana Suárez. ${ }^{3}$
}

\begin{abstract}
Resumen
Objetivo: El propósito de esta revisión bibliográfica fue evaluar la evidencia disponible con respecto al beneficio del uso de pastas fluoradas en la prevención de la Caries de la Infancia Temprana en niños menores de 5 años. Métodos: Se realizó una revisión de literatura en varias bases de datos electrónicas como PubMed, MEDLINE, EBSCO, LILACS, COCHRANE; desde el año 1997 hasta el 2019. Resultados: Un total de 50 artículos cumplieron con los criterios de inclusión y en su mayoría concluyeron que el uso de
\end{abstract}

fluoruros debe incorporarse en los niños debido a la cantidad de beneficios que proporciona. Conclusiones: La caries de la infancia temprana representa un inconveniente sobre todo en países en vías de desarrollo y disminuye considerablemente la calidad de vida del niño y de su familia. Utilizar pastas dentales altas en flúor puede implementarse como medida preventiva para hacer que la prevalencia de esta enfermedad disminuya en niños menores de 5 años.

Palabras clave: fluoruros, lactantes, fluorosis dental.

Artigo da revisao

\section{O uso de fluoretos em crianças menores de 5 anos. Evidência. Revisão bibliográfica.}

\section{Resumo}

Objetivo: $\mathrm{O}$ propósito desta revisão bibliográfica foi avaliar as evidências disponíveis sobre o benefício do uso de pastas fluoretadas na prevenção de cárie precoce em crianças menores de 5 anos de idade. Métodos: Uma revisão de literatura

\footnotetext{
Doctora en Odontología Universidad Central de Venezuela, Especialista en Odontopediatría Universidad Santa María. Profesora del Postgrado de Odontopediatría Universidad de Carabobo. Valencia. Venezuela.

Residente del Postgrado de Odontopediatría Universidad de Carabobo. Valencia. Venezuela.

3. Especialista en Odontopediatría. Universidad Central de Venezuela.
} 
foi realizada em diversas bases de dados eletrônicas, como PubMed, MEDLINE, EBSCO, LILACS, COCHRANE; de 1997 a 2019. Resultados: Um total de 50 artigos preencheram os critérios de inclusão e a maioria concluiu que o uso de fluoretos deve ser incorporado em crianças devido ao montante de benefícios que ele proporciona. Conclusões: A cárie na primeira infância representa uma desvantagem especialmente nos países em desenvolvimento e reduz consideravelmente a qualidade de vida da criança e de sua família. O uso de cremes dentais fluoretados ricos em flúor pode ser implementado como uma medida preventiva para reduzir a prevalência desta doença em crianças menores de 5 anos.

Palavras-chave: fluoretos, lactentes, fluorose dentária.

Article of revision

\section{The use of fluorides in children under 5 years old. Evidence. Bibliographic review.}

\begin{abstract}
Objective: The aim of this literature review was to evaluate the available evidence regarding the benefit of the use of fluorinated pastes in the prevention of early childhood caries in children under 5 years of age. Methods: A literature review was conducted in several electronic databases such as PubMed, MEDLINE, EBSCO, LILACS, COCHRANE from 1997 to 2019. Results: A total of 50 articles met the inclusion criteria and most of them concluded that
\end{abstract}

\section{Introducción}

Desde algunos años las recomendaciones para el uso de pastas dentales en niños menores de 5 años han sufrido cambios. Son muchos los profesionales del área de salud pediátrica que aun no manejan una información consistente ni clara en cuanto a los consejos que deben impartirse a los padres y cuidadores. the use of fluorides should be utilized in children due to the amount of benefits it provides. Conclusions: Early childhood caries represents a handicap especially in developing countries as it considerably reduces the quality of life of the child and his family. Using toothpastes high in fluoride can be implemented as a preventive measure to reduce the prevalence of this disease in children under 5 years.

Key words: fluorides, infants, dental fluorosis.

El papel del fluoruro en la salud bucal se ha estudiado durante muchas décadas. El flúor es uno de los elementos más abundantes en la naturaleza, utilizado ampliamente de forma tópica en el tratamiento de la caries dental por sus propiedades anticariogénicas $\mathrm{y}$ antimicrobianas. ${ }^{1}$

Su uso ha demostrado tener un efecto 
positivo sobre la prevención y se ha catalogado como una de las medidas de salud pública más relevante del siglo XX, aunque su ingesta excesiva tiene efectos adversos sobre el esmalte en desarrollo, ${ }^{2}$ $\mathrm{y}$ antes de los 6 años puede generar un fenotipo hipomineralizado, poroso y de menor dureza. ${ }^{2,3}$

El consumo de fluoruros es necesario para la salud, ya que desempeña un papel importante en el mantenimiento de la estructura y la función fisiológica de los huesos y los dientes. Las principales fuentes de flúor se encuentran en los productos dentales, alimentos y aguas fluoradas. Su concentración en el agua depende de la ubicación geográfica y su ingesta en exceso a altas concentraciones puede causar fluorosis y toxicidad aguda en todo el organismo. ${ }^{4}$

El principal transporte de flúor tópico es la saliva, disminuyendo la desmineralización y aumentando la remineralización del esmalte, siendo clave la frecuencia de la exposición. ${ }^{5}$ Las pastas dentales con fluoruros se han recomendado durante mucho tiempo como parte de las medidas para prevenir la caries dental. Algunos autores señalan que su uso inadecuado puede contribuir a la ingesta total de fluoruro por parte de niños menores de 3 años, siendo una de las razones por las que se han relacionado con el desarrollo de fluorosis dental..$^{6-7}$ Entre los factores que provocan su consumo en exceso están: la ingesta de la pasta dentífrica en los primeros años de vida, el empleo inadecuado de flúor, la reconstitución de la fórmula para lactantes con aguas fluoradas, la ingesta de alimentos y bebidas elaboradas con agua fluorada. ${ }^{5}$
De igual manera, las pastas dentales tienen una función importante en el mantenimiento de la salud bucal, evitando la formación de biopelícula y puliendo la superficie dental, siendo más eficaces las que contienen fluoruros. Sin embargo, los beneficios pueden verse afectados por múltiples factores como su concentración, la cantidad de pasta utilizada en el cepillo dental y las variaciones individuales, incluida la duración y la frecuencia del cepillado y enjuague. ${ }^{1}$

La ingesta de fluoruros durante la primera infancia contribuye al desarrollo de la resistencia a la caries mediante la maduración pre-eruptiva y el mejoramiento de la estructura del esmalte. ${ }^{8}$ Existe actualmente controversia y desconocimiento acerca de la incorporación de pasta fluoradas desde la primera aparición de diente primario en la boca del lactante. Algunas asociaciones internacionales han recomendado su uso por el aumento en la prevalencia de la Caries de la Infancia Temprana (CIT) en niños menores de 5 años.

Los especialistas en odontología pediátrica y cuidadores han recibido información inconsistente en este tópico y existe confusión y falta de credibilidad si el uso de pastas fluoradas es apropiado en lactantes. El objetivo de esta revisión bibliográfica fue evaluar la evidencia disponible con respecto al beneficio del uso de pastas fluoradas en la prevención de la Caries de la Infancia Temprana en niños menores de 5 años.

\section{Material y Método}

Se realizó una revisión de literatura en varias bases de datos electrónicas como 
PubMed, MEDLINE, EBSCO, LILACS, EMBASE; desde el año 1997 hasta el 2019. Los términos utilizados en la búsqueda de la literatura fueron en inglés y español: "fluoride", "toothpaste", "infant", "breastfeeding", "fluorosis". Fluoruros, pastas dentales, lactantes, lactancia materna y fluorosis.

Después de la búsqueda se encontraron 241 artículos, de los cuales solo 53 cumplieron con los criterios de inclusión.

Para esta revisión de literatura los criterios de inclusión fueron: Guías clínicas, revisiones sistemáticas, metaanálisis, estudios observacionales longitudinales, estudios observacionales casos y control, estudios observacionales transeccionales y se excluyeron los reportes de caso.

\section{Resultados:}

Un total de 53 artículos cumplieron con los criterios de inclusión, la mayoría de los últimos 5 años (62\%). La mayor parte de estudios concluyeron que el uso de fluoruros debe incorporarse en los niños debido a la cantidad de beneficios que proporciona.

\section{El flúor y los lactantes}

Aunque los efectos anti-caries del fluoruro estándar $(\mathrm{F})$ en pastas de dientes están bien establecidos, su uso por preescolares (de 2 a 5 años) ha suscitado inquietudes con respecto al desarrollo de fluorosis dental. ${ }^{9}$ La evidencia reciente ha demostrado que las pastas dentales con altas concentraciones de fluoruro mayor de 1500 ppm, pueden ayudar a prevenir y tratar la caries en niños y adolescentes de alto riesgo. ${ }^{10}$
Se ha señalado aumento en la prevalencia de fluorosis dental (principalmente leve) como resultado de una exposición sistémica excesiva al fluoruro, definiendo el periodo crítico para su desarrollo la odontogénesis de dientes primarios desde los 4 meses de vida intrauterina hasta los 11 meses luego del nacimiento. Según ellos la principal fuente de ingesta en bebés menores de 6 meses es la leche materna o leche de fórmula. ${ }^{11,12}$

Nakornchai et al. realizaron un estudio para determinar el exceso de fluoruro durante el cepillado dental comparando la cantidad de orina estimada en bebés y niños antes y después del cepillado, en donde participaron niños menores de 2 años y de 2.5 años. Encontraron que los niños menores de 2 años tuvieron un aumento significativo de la excreción estimada de fluoruro en orina después del cepillado con una cantidad de pasta dental del tamaño de un guisante, pero en niños de 2 a 2.5 años de edad no hubo aumento de la excreción urinaria después del cepillado con una cantidad del tamaño de un guisante, utilizándose una concentración de 1000 ppm de pasta dental fluorada. ${ }^{13}$

Describiendo su biodisponibilidad, aproximadamente el $90 \%$ del fluoruro diario ingerido se absorbe en el tracto digestivo. La proporción de elemento ingerido retenido en el cuerpo es de aproximadamente $55 \%$ en niños y $36 \%$ en adultos, y el resto absorbido se excreta a través de los riñones. Aproximadamente el $99 \%$ del fluoruro en el cuerpo está asociado con tejidos calcificados y está disponible para el esmalte durante el período de odontogénesis o maduración pre-eruptiva. La absorción a través de la mucosa bucal es limitada y probablemente 
representa menos de $1 \%$ de la ingesta diaria, pero el fluoruro afecta la superficie exterior del esmalte cuando está en la cavidad bucal. ${ }^{14}$ La biodisponibilidad del fluoruro en los lactantes es mayor, por lo tanto, es necesario un estándar exacto del consumo de flúor. Sin embargo, el estilo de vida y hábitos son diferentes en cada país y deben tomarse medidas según cada condición. ${ }^{8,15}$ La eliminación a través del riñón es menor entre los niños que en los adultos. Por lo tanto, la alta ingesta durante la infancia mejora la retención de más fluoruro. ${ }^{16}$

El agua fluorada de manera óptima puede aumentar el riesgo de fluorosis en pacientes menores de seis meses. Determinar las estimaciones de la ingesta de fluoruro y la exposición es especialmente importante para bebés y niños con dientes en desarrollo. ${ }^{12} \mathrm{La}$ mayoría de las aguas embotelladas tienen un contenido promedio de fluoruro de menos de 0.01 ppm, mientras que aguas embotelladas / purificadas han mostrado un contenido promedio de fluoruro de menos de 0.03 ppm. Los sistemas que involucran ósmosis inversa también dan como resultado concentraciones de un fluoruro subóptimo. En consecuencia, es posible que algunos bebés no estén recibiendo el máximo beneficio del flúor para la prevención de caries. ${ }^{17} \mathrm{Sin}$ embargo, la Asociación Dental Americana recomienda que se continúe la lactancia materna durante el primer año de vida y que la fórmula (uso infantil) sea reconstituida con agua que esté libre de fluoruro o tenga bajas concentraciones de fluoruro cuando el riesgo potencial de fluorosis del esmalte sea alto. ${ }^{18}$ De igual forma, hay que tener en cuenta que la temperatura ambiental afecta la ingesta de agua y por ende el consumo de flúor. ${ }^{19}$
Debido a la creciente preocupación por la mayor cantidad de ingesta de fluoruro en niños pequeños de múltiples fuentes y mayor prevalencia de fluorosis del esmalte, la Asociación Dental Americana (ADA) ofreció directrices provisionales sobre la fórmula infantil y las dosis de flúor para lactantes en 2007. ${ }^{20}$

\section{Lactancia materna y Caries de la Infancia Temprana}

A medida que el niño crece y recibe lactancia materna se observan grandes beneficios de la misma en el desarrollo tanto del que la recibe como para la madre. Sin embargo, también se ha encontrado que en niños menores de 5 años ahora hay mayores reportes de caries dental. Esto llama la atención a estudiar el por qué de esta relación. La Caries de la Infancia Temprana (CIT) es la enfermedad crónica infantil más prevalente en el mundo y afecta negativamente la salud bucal en todos los aspectos socioeconómicos. Pero sobre todo en niveles socioeconómicos bajos. La falta de salud bucal en la primera infancia o infancia temprana es un factor de riesgo ya que generalmente continúa durante todo el curso de la vida. ${ }^{21,22}$

La prevalencia de CIT difiere según el grupo examinado, $y$ se ha informado una prevalencia de hasta el $85 \%$ para los grupos desfavorecidos. La CIT ha sido definida como la presencia de uno o más dientes primarios cariados, faltantes $\mathrm{o}$ restaurados en niños de 71 meses ( 5 años) o menos. ${ }^{23}$ En múltiples estudios, se ha demostrado que la caries dental tiene un impacto social importante en los niños con respecto a su dolor, sueño, actividades sociales, alimentación y asistencia a la escuela, así como un impacto emocional durante la infancia. ${ }^{24}$ 
Las revisiones sistemáticas publicadas muestran cierta evidencia de un efecto protector de la lactancia materna contra la maloclusión de la dentición primaria, pero no hay evidencia de apoyo para la dentición mixta y maloclusiones de la dentición permanente. Con respecto a la caries dental, los estudios reportan un beneficio de la lactancia materna hasta los 12 meses, pero una asociación positiva entre la caries y la lactancia cuando se prolonga su duración después de los 12 meses de edad y durante las tomas nocturnas. ${ }^{25}$ Existe también evidencia que asocia la presencia de caries dental en la infancia con la disminución de la calidad de vida del niño y de su familia. ${ }^{26} \mathrm{~A}$ pesar de alguna evidencia sobre el mayor riesgo de caries dental en niños amamantados más allá de los 12 meses de edad, estos hallazgos se derivan de estudios altamente heterogéneos y la falta de control es uno de los principales factores de confusión. ${ }^{27}$

La leche materna tiene una composición nutricional y una biodisponibilidad superior. Sin embargo, los azúcares proporcionan aproximadamente el $40 \%$ de la energía en la leche materna madura. Esta última contiene más azúcares que la leche bovina, aproximadamente el $7 \%$ en comparación con un $4,8 \%$. La leche materna también es significativamente más baja en calcio y fosfato; factores que protegen contra la caries dental, en comparación con la leche bovina. ${ }^{25}$ Los estudios in vitro reportan que la leche materna puede reducir el $\mathrm{pH}$ de la biopelícula y causar mayor disolución del esmalte en comparación con la leche bovina. ${ }^{28}$

La leche humana produce más caries que la leche de vaca en superficies lisas, pero exhibe menor cariogenicidad que la fórmula infantil o la sacarosa. La alta concentración de lactosa encontrada en la leche humana tiene el potencial de reducir el $\mathrm{pH}$ de la biopelícula, llevando a caries dental. ${ }^{29}$

La OMS sugiere "mantener como patrón fundamental la lactancia materna exclusiva hasta los 6 meses, y luego, junto con otros alimentos, hasta los 2 años de vida", pero hay países lejanos a esta meta. A escala mundial, tanto en los países en vías de desarrollo como en los desarrollados, se incrementa el abandono de la lactancia materna exclusiva, que es sustituida por la artificial o la mixta. ${ }^{21}$

La alta frecuencia diaria de lactancia materna ha sido asociada con CIT y CIT severa, incluso después del ajuste de la ingesta total de carbohidratos y frecuencia de uso de biberones. ${ }^{30}$ Una revisión sistemática encontró que la asociación de la lactancia materna y CIT más allá de los 12 meses de edad fue más fuerte cuando la lactancia fue frecuente o nocturna. ${ }^{31}$

Feldens et al. reportaron una asociación prospectiva entre la frecuencia de alimentación a los 12 meses de edad, incluida la lactancia materna y el uso de biberones de alta frecuencia, y caries dental a la edad de 3 años. Estos hallazgos sugieren que la prevención de la caries dental podría lograrse haciendo hincapié en que el niño amamantado o alimentado con biberón debe ser cuidado a intervalos menos frecuentes una vez que se hayan introducido alimentos complementarios nutricionalmente suficientes. La limitación de la frecuencia de otros bocadillos o bebidas también puede resultar en la prevención de la caries. ${ }^{32}$

Al comparar niños con uso poco frecuente de biberón y la lactancia materna a los 
12 meses, a los 38 meses, la prevalencia de CIT fue 1.8 veces mayor en los niños amamantados más de tres veces al día, 1.4 veces más en los niños alimentados con biberón más de tres veces/día y 1.5 veces mayor con alta frecuencia combinada de biberón y amamantamiento juntos. ${ }^{33}$ Los niños amamantados más allá de 12 meses, un tiempo durante el cual todos los dientes primarios erupcionan, tienen un mayor riesgo de caries. Esto puede ser debido a otros factores que están vinculados con lactancia prolongada incluyendo alimentación nocturna durante el sueño, alimentos/bebidas cariogénicas en la dieta o prácticas de higiene bucal inadecuadas. ${ }^{31}$ Ser amamantado durante $\geq 24$ meses aumenta el riesgo de tener caries dental a los 5 años de edad. Del mismo modo, aquellos niños eran más propensos a desarrollar CIT severa. Estos efectos no fueron medidos por el consumo de azúcar durante el curso de vida. ${ }^{27}$

La introducción de las fuentes de alimentos a los bebés debe ser baja en carbohidratos simples (azúcares) e implementar la higiene bucal. Las prácticas deben comenzar con la erupción del primer diente para que la biopelícula se elimine de las superficies dentales para reducir el riesgo de caries dental. ${ }^{34,35}$

Un estudio reciente sugirió que la diversidad genética de Streptococcus mutans, la bacteria más comúnmente asociada con el desarrollo de la caries dental, puede estar asociado con la susceptibilidad a la caries en aquellos niños que presentan tales bacterias. Sin embargo, la presencia de la lactancia materna prologada se mantuvo asociada a CIT severa a pesar de la presencia de $S$. mutans. ${ }^{36}$

Toda esta evidencia nos ayuda a tener una idea clara de que la CIT se desarrolla más de lo que muchos pensamos. Y que el factor protector de la lactancia materna es más hacia el desarrollo de los maxilares durante los primeros 12 meses de vida.

\section{Discusión}

Es muy interesante la conclusión a la que llegaron Santos et al. en su estudio evaluando los efectos de las pastas dentales bajas en $\mathrm{F}(<600$ ppm) y estándar F $(1,000-$ 1,500 ppm) en la prevención de caries en la dentición primaria y fluorosis estéticamente objetable (moderada a severa) en la dentición permanente por medio de una revisión sistemática de ensayos clínicos y metaanálisis. Las pastas dentales bajas en $\mathrm{F}$ aumentaron significativamente el riesgo de caries en los dientes primarios y no disminuyeron significativamente el riesgo de fluorosis estéticamente objetable en los dientes permanentes anteriores superiores. No hay evidencia para apoyar el uso de pastas de dientes de bajo $\mathrm{F}$ en preescolares con respecto a la prevención de caries y fluorosis dental. ${ }^{9}$

Pocas pastas dentales destinadas a niños presentan concentraciones de fluoruro solubles capaces de prevenir la caries dental. Es necesario implementar medidas reglamentarias para la comercialización de pastas dentales destinadas con al menos 1000 ppm de fluoruro soluble, especialmente en lugares sin otras fuentes como el agua para la población. ${ }^{37}$

En los niños en edad preescolar se ha encontrado que durante el cepillado tragan una cantidad significativa de pasta dental ${ }^{38}$ y la ingesta es mayor en los niños más pequeños. ${ }^{37}$ Por lo tanto, se debe usar una pequeña cantidad de pasta dental F estándar bajo la supervisión de los padres y realizar 
un cepillado dental después de las comidas para disminuir la biodisponibilidad de F. ${ }^{39}$

También hay evidencia científica limitada con respecto a la efectividad y el riesgo de fluorosis asociada con utilizando diferentes cantidades de pasta dental con fluoruro (frotis versus tamaño de guisante) en niños menores de 3 años. Sin embargo, los resultados de Wright et al. sugieren que las pastas dentales con 500 ppm de fluoruro o más tienen un mayor efecto en la reducción de caries en comparación con las pastas dentales por debajo de esta concentración en niños de 6 años o menos. Ingerir cantidades del tamaño de un guisante o más puede conducir a una fluorosis leve. ${ }^{40}$

Una proporción relativamente grande de la ingesta de fluoruro es retenida en el cuerpo en lactantes destetados. Esta es una consideración importante en los programas de prevención, con metas para maximizar la prevención de caries minimizando el riesgo de fluorosis dental. ${ }^{11}$ Sin embargo, también es necesario resaltar que la fluorosis no es una enfermedad mortal, y es posible prevenirla y tratarla. Los índices de fluorosis (como Índice de Dean, el índice Thylstrup y Fejerskov o el índice de riesgo de fluorosis) si se usan solos, pueden dar como resultados un diagnóstico erróneo de fluorosis dental y estar más relacionado a defectos del esmalte. Existe una necesidad de datos precisos de fluorosis dental, la inconsistencia de los informes sobre la prevalencia de fluorosis sugiere que las definiciones deberían ser más precisas. Se necesitan métodos de diagnóstico para detectar fluorosis dental y distinguirla de otros defectos de esmalte ${ }^{41}$ como hipoplasias aisladas, amelogénesis imperfecta, hipomineralización incisivo molar.

La caries dental es un problema de salud pública porque es una condición importante pero prevenible que lleva a dolor y sufrimiento que afecta la calidad de vida de los niños. El conocimiento de los padres de higiene y prevención bucal sobre el uso de fluoruro en niños es insuficiente, ya que la mayoría no están familiarizados con el tipo de pasta de dientes que deben usar sus hijos, ni son conscientes de la cantidad adecuada de dentífricos para aplicar en el cepillo de dientes. Por lo tanto, es imperativo que los padres controlen el cepillado dental de los niños, especialmente cuando están en edades tempranas, con el fin de evitar toxicidad de la pasta dental con flúor. ${ }^{41,42}$

La Academia Americana de Pediatría (ADA) recomienda pasta dental fluorada a todos los niños que comienzan con la erupción dental, independientemente del riesgo de caries. Además, se recomienda el barniz de fluoruro para todos los niños cada 3 a 6 meses desde la primera erupción dental hasta que terminen su erupción. Es posible adherirse a las pautas de barniz de fluoruro oral para todos los niños menores de 3 años en una práctica de atención primaria. ${ }^{43}$ Sin embargo, es de destacar que las aplicaciones tópicas de 0,1 ml de barniz de fluoruro aumentaron significativamente la excreción de fluoruro. ${ }^{44} \mathrm{~A}$ pesar de que el barniz de flúor ha mostrado un efecto anticaries modesto en los preescolares. ${ }^{45}$ Se necesitan análisis de costo-efectividad para evaluar si los servicios dentales deben adoptar o abandonar su uso. ${ }^{46}$

Algunos países como Perú, tienen pastas dentales fluoradas, sin embargo, al estudiarlas solo el 53\% contenía una concentración de fluoruro soluble total (TSF) mayor de 1000 ppm F, la concentración mínima necesaria para proporcionar un efecto anticaries. ${ }^{47}$ Aunque en Brasil se evaluaron las concentraciones de fluoruro 
total (TF) y fluoruro soluble total (TSF) en las pastas de dientes brasileñas más vendidas. Estas presentaron la concentración de fluoruro disponible para controlar las caries independientemente de la región donde se compran. ${ }^{48}$ La cantidad de fluoruros en las pastas varía de acuerdo a cada país. ${ }^{49}$

Los cuidados de salud bucal deben ser garantizados para que se mantenga un equilibrioentremaximizar el efecto protector contra la caries dental y minimizar el riesgo de fluorosis dental. Es necesario elevar la conciencia de los padres, implementando programas educativos adecuados que lleguen a toda la población. ${ }^{42}$ La exposición al fluoruro, ciertos hábitos dietéticos, e higiene bucal pueden influir en el efecto de la lactancia materna en caries dental, particularmente considerando sus efectos a largo plazo. ${ }^{33,50}$

Actualmente se han implementado políticas para reducir la exposición excesiva al fluoruro durante el período de desarrollo del diente, que tuvieron éxito en la reducción de la fluorosis dental sin comprometer la prevención de caries. Ahora hay una comprensión mucho mejor de la percepción pública de la fluorosis dental, y la fluorosis leve no es un problema estético. Las ventajas de la fluoración del agua son que proporciona una importante prevención de caries durante toda la vida, es económica y reduce las desigualdades en la salud. Las pastas dentales que contienen fluoruro son, la forma más importante de administrar el efecto beneficioso del fluoruro en todo el mundo. Los efectos preventivos de la exposición conjunta (por ejemplo, el uso de pasta dental con fluoruro en un área fluorada) son aditivos. La Organización Mundial de la Salud ha informado a los estados miembros sobre los beneficios su uso apropiado. Muchos países tienen políticas para maximizar sus beneficios, pero muchos aún no lo han hecho. ${ }^{51}$

\section{Conclusión}

Se concluye por medio de esta revisión bibliográfica que, aunque se necesita mayor evidencia científica para soportar los beneficios y riesgos del uso de fluoruros en lactantes y niños menores de 5 años, se deben hacer cambios en las medidas impartidas. La caries de la infancia temprana representa un inconveniente sobre todo en países en vías de desarrollo y disminuye considerablemente la calidad de vida del niño y de su familia. Utilizar pastas dentales altas en flúor, específicamente mayores a 1000 ppm desde que aparece el primer diente en boca, puede implementarse como medida preventiva para hacer que la prevalencia de esta enfermedad disminuya en niños menores de 5 años. También deben proponerse como medidas de salud pública y que toda la población reciba información actualizada conociendo los riesgos y beneficios que conllevan.

\section{Referencias bibliográficas}

1. Ullah R, Zafar M. Oral and dental delivery of fluoride: a review. Research review. 2015; 48(3): 195-204.

2. Rubio G, Martignon S, Parra J, Naranjo W. Patogénesis de la fluorosis dental: mecanismos bioquímicos y células. Revista Facultad de Odontología Universidad de Antioquia. 2017; 28(2). Disponible en: https:// www.researchgate.net/publication/319306335_Pathogenesis_Of_Dental_Fluorosis_Biochemical_And_ Cellular_Mechanisms

3. López N, ZaragoziI E, Gonzalez L. Efectos de la ingestión prolongada de altas concentraciones de fluoruros. Revista 6 de abril. 2015; 54(260): 83-94.

4. Zuo H, Che L, Kon M, Qi L, Lu P, Wu P, Yang Y, Chen K. Toxic effects of fluoride on organisms. Life Scie. 2018; 198: 18-24. 
5. Calderón J, Lopez N, Dobarganes AM. Caracteristicas generales de la fluorosis dental. Revista electrónica Dr. Zoilo E. Marinello. 2014; 39(12). Disponible en: http://revzoilomarinello.sld.cu/index.php/zmv/article/ view/128

6. Buzalaf MAR, Levy SM. Fluoride intake of children: considerations for dental caries and dental fluorosis. Monogr Oral Sci. 2011;22:1-19.

7. Levy SM, Hillis SL, Warren JJ, Broffitt BA, Mahbubul Islam AK, Wefel JS, Kanellis MJ. Primary tooth fluorosis and fluoride intake during the first year of life. Community Dent Oral Epidemiol. 2001; 30(4):286-95.

8. Yanagida R, Satou R, Sugihara N. Estimation of daily fluoride intake of infants using the microdiffusion method. J Dent Scien. 2019; 14:1-6.

9. Santos APP, Oliveira BH, Nadanovsky P. Effects of Low and Standard Fluoride Toothpastes on Caries and Fluorosis: Systematic Review and Meta-Analysis. Caries Res. 2013;47: 382-90.

10. Pretty LA. High fluoride concentration toothpastes for children and adolescents. Caries res. 2016; 50(1): 9-14.

11. Zohoori FV, Omid N, Sanderson RA, Valentine RA, Maguirre A. Fluoride retention in infants living in fluoridated and non-fluoridated areas: effectos of weaning. Br J Nutr. 2019;121(1):74-81.

12. Harriehausen C, Dosani F, Chiquet B, Barratt M, Quock R. Fluoride intake of infants from formula. J Clin Pediatr Dent. 2019; 43(1):34-41.

13. Nakorncha S, Horsophonphong S, Sanguansin S, Surarit R. Estimation of urinary fluoride excretion in infants and toddlers after brushing with fluoride toothpaste. Trop Med Public Health. 2018; 49(5):909-15.

14. O'Mullane D, Baez R, Jones S, Lennon MA, Petersen PE, Rugg-Gunn AJ, Whelton H, Whitford GM. Fluoride and oral health. Community Dent Health 2016; 33(2):69-99.

15. Ryosuke Y, Ryouichi S, Naoki S. Estimation of daily fluoride intake of infants using the microdiffusion method. J Dent Sci. 2019; 14:1-6.

16. Bannari Amman, Sathyamangalam Tamil. Contribution of infant formula and tea on daily fluoride intake and prevalence of fluorosis among infants and children. J Pub Health Dent. 2000;60(3):131-9.

17. Quock R, Chan J. Fluoride content of bottled water and its implications for the general dentist. Gen Dent. 2009; 57(1):29-33.

18. Berg J, Gerweck C, Hujoel P, et al. Evidence-based clinical recommendations regarding fluoride intake from reconstituted infant formula and enamel fluorosis: a report of the American Dental Association council on scientific affairs. J Am Dent Assoc. 2011; 142:79-87.

19. Lima-Arsati YBO, Gomes ARLF, Santos HKA, Arsati F, Oliveira MC, Freitas VS. Exposure to fluoride of children during the critical age for dental fluorosis, in the semiarid region of Brazil. Cien Saude Colet. 2018;23(4):1045-54.

20. American academy of pediatric dentistry. Guideline on fluoride therapy. Reference manual 2014-15. Pediatr Dent 2014; 34(6): 171-4.

21. Kassebaum N, Smith A, Bernabé E , Fleming T, Reynolds A, Vos T , Murray C, Marcenes C, GBD 2015 Oral Health Collaborators. Global, regional, and national prevalence, incidence, and disability-adjusted life years for oral conditions for 195 countries, 1990-2015: a systematic analysis for the global burden of diseases, injuries, and risk factors. J Dent Res. 2017; 96(4):380-7.

22. Broadbent J, Thomson $\mathrm{W}$, Ramrakha S, et al. Community water fluoridation and intelligence: prospective study in New Zealand. Am J Public Health. 2015; 105(1):72-6

23. Anil S, Anand PS. Early Childhood Caries: Prevalence, Risk Factors, and Prevention. Front Pediatr. 2017 ;5:157.

24. Gilchrist F, Marshman Z, Deery C, Rodd HD. The impact of dental caries on children and young people: what they have to say? Int J Paediatr Dent. 2015;25(5):327-38.

25. Peres KG, Chaffee BW, Feldens CA, Flores-Mir C, Moynihan P, Rugg-gunn A. Breastfeeding and oral health: evidence and methodological challenges. J Dent Res 2018;97(3):251-8.

26. Kramer PF, Feldens CA, Ferreira SH, Bervian J, Rodrigues PH, Peres MA. Exploring the impact of oral diseases and disorders on quality of life of preschool children. Community Dent Oral Epidemiol. 2013; 41(4): 327-35.

27. Peres KG, Nascimento GG, Peres MA, Mittinty MN, Demarco FF, Santos IS, Matijasevich A, Barros AJD. Impact of Prolonged Breastfeeding on Dental Caries: A Population-Based Birth Cohort Study. Pediatrics. 2017; 140(1):e2016294.

28. Rugg-gunn AJ, Roberts GJ, Wright WG. Effect of human milk on plaque ph in situ and enamel dissolution in vitro compared with bovine milk, lactose, and sucrose. Caries Res. 1985; 19(4):327-34. 
29. Prabhakar AR, Kurthukoti AJ, Gupta P. Cariogenicity and acidogenicity of human milk, plain and sweetened bovine milk: an in vitro study. J Clin Pediatr Dent. 2010;34(3):239-47.

30. Mohebbi SZ, Virtanen JI, Vahid-Golpayegani M, Vehkalahti MM. Feeding habits as determinants of early childhood caries in a population where prolonged breastfeeding is the norm. Community Dent Oral Epidemiol. 2008; 36(4): 363-9.

31. Tham R, Bowatte G, Dharmage SC et al. Breastfeeding and the risk of dental caries: a systematic review and meta-analysis. Acta Paediatr 2015 104: 62-84.

32. Feldens CA, Rodrigues PH, de Anastacio G, Vitolo MR, Chaffee BW. Feeding frequency in infancy and dental caries in childhood: a prospective cohort study. Int Dent J. 2018;68(2):113-21.

33. Feldens CA, Giugliani ER, Vigo A, Vítolo MR. Early feeding practices and severe early childhood caries in four-year-old children from southern Brazil: a birth cohort study. Caries Res. 2010;44(5):445-52.

34. Valaitis R, Hesch R, Passarelli C, Sheehan D, Sinton J. A systematic review of the relationship between breastfeeding and early childhood caries. Can J Public Health 2000; 91: 411-7.

35. White V. Breastfeeding and the risk of early childhood caries. Evid Based Dent 2008; 9: 86-8.

36. Yu LX, Tao Y, Qiu RM, Zhou Y, Zhi QH, Lin HC. Genetic polymorphisms of the sortase A gene and socialbehavioural factors associated with caries in children: a case-control study. BMC Oral Health. 2015;15:54

37. Santos N, Monteiro R, Santos L, Vasconcelos S, Ribeiro F, Andrade D. Identification and analysis of the fluoride concentration in toothpastes intented for children. Pesqui Bras Odontopediatria Clin Integr. 2019; 19(1):e3986.

38. Franco AM, Martignon S, Saldarriaga A, Gonzalez MC, Arbelaez MI, Ocampo A, Luna LM, Martinez-Mier EA, Villa AE: Total fluoride intake in children aged 22-35 months in four Colombian cities. Community Dent Oral Epidemiol 2005; 33:1-8.

39. Cury JA, Del Fiol FS, Tenuta LM, Rosalen PL: Low-fluoride dentifrice and gastrointestinal fluoride absorption after meals. J Dent Res 2005;84: 1133-7.

40. Wright T, Hanson N, Ristic H, Whall CW, Estrich C, Zentz R. Fluoride toothpaste efficacy and safety in children younger than 6 years A systematic review. JADA 2014;145(2):182-9

41. Sabokseir A, Golkari A, Sheiham A. Distinguishing between enamel fluorosis and other enamel defects in permanent teeth of children. Peer J. 2016: 2-11.

42. Turska A, Swiatkowska M, Walczak M, Olczak D. What do parents know about the use of fluoride products in children? a questionnaire study. Research Report. 2018; 51(2):114-21.

43. Sudhanthar S, Lapinski J, Turner J, Gold J, Sigal Y, Thakur K, Napolova O, Stiffler M. Improving oral health through dental fluoride varnish application in a primary care paediatric practice. BMJ Open Qual. 2019;8(2):e000589.

44. Lockner F, Twetman S, Stecksen-Blicks C. Urinary fluoride excretion after application of fluoride varnish and use of fluoride toothpaste in young children. Int J Paediatr Dent. 2017;27(6):463-8.

45. Carvalho DM, Salazar M, Oliverira BH, Coutinho ES. Fluoride varnishes and decrease in caries incidence in preschool children: a systematic review. Res Bras Epidemiol. 2010;13(1):139-49.

46. Sousa FSO, Santos APP, Nadanovsky P, Hujoel P, Cunha-Cruz J, Oliveira BH. Fluoride Varnish and Dental Caries in Preschoolers: A Systematic Review and Meta-Analysis. Caries Res. 2019; 20:1-12.

47. Chavez BA, Vergel GB, Cáceres CP, Perazzo MF, Vieira-Andrade RG, Cury JA. Fluoride content in children's dentifrices marketed in Lima, Peru. Braz Oral Res. 2019;33: e051.

48. Ricomini Filho AP, Tenuta LM, Fernandes FS, Calvo AF, Kusano SC, Cury JA. Fluoride concentration in the top-selling Brazilian toothpastes purchased at different regions. Braz Dent J. 2012;23(1):45-8.

49. Cochran JA, Ketley CE, Duckworth RM, van Loveren C, HolbrookWP, Seppa L, Sanches L, Polychronopoulou A, O'Mullane DM. Development of a standardized method for comparing fluoride ingested from toothpaste by 1.5-3.5-year-old children in seven European countries. Part 2. Ingestion results. Community Dent Oral Epidemiol. 2004;32(suppl 1): 47-53.

50. Thitasomakul S, Piwat S, Thearmontree A, Chankanka O, Pithpornchaiyakul W, Madyusoh S. Risks for early childhood caries analyzed by negative binomial models. J Dent Res. 2009;88(2):137-41.

51. Whelton HP, Spencer AJ, Do LG, Rugg-Gunn AJ. Fluoride Revolution and Dental Caries: Evolution of Policies for Global Use. J Dent Res. 2019;98(8):837-46.

Recibido: 19/07/19

Aceptado: 05/10/19

Correspondencia: María Gabriela Acosta de Camargo, e-mail: gabrieladecamargo@yahoo.com 